\title{
APLICAÇÃO DAS TÉCNICAS DE APRENDIZAGEM ATIVA EM CURSOS DE ENGENHARIA
}

DOI: 10.37702/2175-957X.COBENGE.2021.3490

Edson Pedro Ferlin - eferlin@live.com

CF ABENGE e UniDomBosco

Rua David Martins Lass 94

82025-190 - Curitiba - PR

Resumo: Este artigo apresenta a aplicação de algumas técnicas de aprendizagem ativa em cursos de engenharia, em específico nos cursos de Engenharia da Computação e Engenharia da Produção, e que poderiam ser utilizadas nos outros cursos, mas não somente de engenharia. A aprendizagem ativa é um elemento catalizador no processo de ensino e aprendizagem e a sua utilização pode resultar em uma melhoria do aprendizado por parte estudante, pois ele é o elemento ativo no processo, deixando de ser um mero expectador e passando a ser o ator principal. Há diversas técnicas que podem ser utilizadas e cada uma delas tem suas características e cabe ao professor analisar e utilizar determinada técnica que possibilite o desenvolvimento das competências nos estudantes. A conclusão reitera a importância de despertar a motivação nos estudantes e colocá-los como elementos ativos no processo de ensino e aprendizagem.

Palavras-chave: Engenharia, Educação, Aprendizagem Ativa, Níveis de Aprendizagem 


\section{APLICAÇÃO DAS TÉCNICAS DE APRENDIZAGEM ATIVA EM CURSOS DE ENGENHARIA}

\section{INTRODUÇÃO}

Uma forma de motivar e cativar os estudantes é colocá-los no centro do processo de ensino e aprendizagem, em que eles são os agentes ativos do processo. Entretanto, isto não pode estar somente no papel, mas deve se traduzir em ações concretas, em que os estudantes devam se comprometer ao longo do processo.

Esta forma de atuar deve estar alinhada ao Projeto Pedagógico do Curso (PPC), bem como com o Plano de Desenvolvimento Institucional (PDI), pois isto afeta a maneira pela qual as disciplinas e os professores interagem e, também, na maneira com a qual os estudantes se envolvem no processo de ensino e aprendizagem.

\section{NÍVEIS DE APRENDIZAGEM}

A Taxonomia dos Objetivos Educacionais de Bloom (BLOOM, 1956), revisada por Anderson \& Krathwohl (2001), define uma hierarquia de seis níveis para o processo cognitivo. Nessa estrutura cada nível representa uma capacidade cognitiva e são organizadas em ordem crescente de complexidade. Nesse modelo tem-se as capacidades: lembrar (nível factual), entender (ser capaz de lidar com conceitos), aplicar (nível procedimental), analisar, avaliar e criar, em que as três últimas capacidades pertencem ao nível mais alto da classificação, o nível metacognitivo.

Conforme destacado em Portilho (2009): "uma das metas do processo de aprendizagem e ensino é estimular o aprendiz a ser autônomo, isto é, sujeito do seu próprio aprender".

Espera-se que dos estudantes de cursos de graduação, em nosso caso particular os estudantes de um curso de engenharia, seja alcançado o nível metacognitivo durante o processo ensino e aprendizagem, como abordado em Pilla \& Ferlin (2010).

\section{APRENDIZAGEM ATIVA}

O termo "aprendizagem ativa" começou a ser utilizado pelo professor inglês Reginald "Reg" William Revans na década de 1940 (REVANS, 2011). Nesse sentido, fazendo um breve apanhado das definições do termo, pode-se definir a aprendizagem ativa como todo tipo de atividade que vai além do mero "escutar", isto é, ler, escrever, discutir ou trabalhar na resolução de problemas. Ativa porque a audição, principalmente quando considerada a tradicional transmissão do conhecimento por meio de aulas expositivas, é uma atividade que "pouco" exige mentalmente dos estudantes. Além disso, é difícil saber se a atenção do "aprendiz" estaria voltada ou não para aquilo que está sendo ouvido.

Conforme Bloom (1956), a aprendizagem ativa deve engajar os estudantes em atividades reflexivas de ordem superior. As atividades reflexivas estão no nível metacognitivo, e são aquelas que exigem a elaboração do pensamento: análise, síntese e avaliação. Elas correspondem a uma constante reflexão sobre a prática, sobre a adequação à realidade do que estiver sendo aprendido. 
A aprendizagem ativa e seus diversos métodos são uma mistura de práticas novas e antigas, como os descritos em Elmor et al. (2019) e Yee (2010). Ainda que os professores não usassem abertamente este termo ou nem mesmo tivessem consciência de que estavam aplicando a aprendizagem ativa, se for realizado um retrospecto sobre as atividades educativas realizadas durante o processo de formação, formal ou não, percebe-se que, em diversos momentos, muitas atividades se enquadram em algum dos exemplos citados na literatura como trabalhos em grupo, trabalhos de pesquisa, seminários, estudo de caso e etc.

Segundo Pinto et al. (2012) há necessidade de "envolver o estudante enquanto protagonista de sua aprendizagem,desenvolvendo ainda o senso crítico diante do que é aprendido, bem como competências para relacionar esses conhecimentos ao mundo real'.

Assim, aprendizagem ativa ocorre quando o aluno interage com o assunto em estudo - ouvindo, falando, perguntando, discutindo, fazendo e ensinando - sendo estimulado a construir o conhecimento ao invés de recebê-lo de forma passiva do professor. Em um ambiente de aprendizagem ativa, o professor atua como orientador, supervisor, facilitador do processo de aprendizagem, e não apenas como fonte única de informação e conhecimento (BARBOSA \& MOURA, 2013, p.55).

\subsection{Aprendizagem Baseada em Problemas (Problem-Based Learning - PBL)}

O Problem-based learning (PBL), ou aprendizagem baseada em problemas, é uma técnica de ensino e aprendizagem que foi implantada inicialmente na faculdade de Medicina da Universidade canadense McMaster, na década de 1960. Uma característica marcante de processos que envolvem PBL é que neles os estudantes tornam-se responsáveis por definir os objetivos educacionais, os meios que vão utilizar, o que vão aprender e com o que vão trabalhar (BARRET, 2004).

Os conceitos fundamentais para PBL, difundidos por Center for Teaching and Learning da Stanford University (STANFORD, 2001), são: a) apresentação inicial de problemas do tipo "precariamente estruturados" ao invés de totalmente pré-especificados; b) ensino centrado no estudante ao invés de centrado no professor; c) trabalhos em grupos ao invés de individualizados; d) professores como facilitadores ao invés de disseminadores de conhecimentos.

Alguns fundamentos práticos do PBL, destacados em Melo (2013) são: a) os problemas expostos aos estudantes devem ser, de preferência, "precariamente estruturados" (ill-structured problems), que são os que permitem que se desenvolva diversas soluções potenciais, autênticos (reais), ou seja, devem ser os problemas enfrentados pelos profissionais; b) os estudantes devem ser envolvidos em práticas e problemas autênticos, sendo obrigados a desenvolverem ações autênticas para solucioná-los; c) os estudantes devem trabalhar em pequenos grupos (em torno de cinco membros; nunca além de oito); d) os grupos devem ser liderados/orientados por um tutor/facilitador dos trabalhos, que deve fornecer ao grupo estratégias de aprendizagem e nunca ser um fornecedor de soluções prontas; e) os grupos devem ser interdisciplinares, contando, se possível, com colaboradores de outras áreas de conhecimentos interrelacionadas.

Em Ribeiro (2008) são apresentados os quatro modelos de PBL em função dos contextos de ensino-aprendizagem. 


\subsection{Estudo de Caso}

O estudo de caso envolve a abordagem de conteúdo por intermédio do estudo de situações de contexto real, as quais são denominadas "casos". Esse modelo pressupõe a participação ativa dos estudantes na resolução de questões relativas ao caso, normalmente em um ambiente colaborativo com seus pares. Apesar de poder ser resolvido individualmente, uma das maiores riquezas dessa abordagem de ensino é a interação que promove mudanças significativas na sala de aula com o trabalho em equipe. Trata-se de uma abordagem ativa e colaborativa, que promove o desenvolvimento da autonomia e da metacognição (PORTILHO, 2009), quando conduzido de forma apropriada.

Segundo Yin (2015), o uso do estudo de caso é adequado quando se pretende investigar o como e o porquê de um conjunto de eventos. $O$ autor ressalta que o estudo de caso é uma investigação empírica que permite o estudo de um fenômeno dentro de seu contexto real, especialmente quando os limites entre o fenômeno e o contexto não estão claramente definidos.

Os casos são construídos em torno de objetivos de aprendizagem (habilidades e competências) que se pretende desenvolver, e são seguidos de questões que devem ser respondidas pelos estudantes no decorrer do desenvolvimento. A presença dessas questões torna o estudo de caso uma abordagem de ensino guiada.

Alguns propósitos dos estudos de caso são apontados por Gil (2007): 1) explorar situações da vida real cujos limites não estão claramente definidos; 2) preservar o caráter unitário do objeto estudado; 3) descrever a situação do contexto em que está sendo feita uma determinada investigação; 4) formular hipóteses ou desenvolver teorias e 5) explicar as variáveis causais de determinado fenômeno em situações complexas que não permitam o uso de levantamentos e experimentos.

\subsection{Mapa Mental}

Os Mapas Mentais são formas de registrar e estruturar as informações de forma gráfica. Segundo Buzan (1996), o criador desta técnica conhecida como Mind Maps (em inglês), são ferramentas de pensamento que permitem refletir exteriormente o que se passa na mente. É uma forma de organizar os pensamentos e utilizar ao máximo as capacidades mentais. Ao analisar um mapa mental, é possível verificar diversas ideias a respeito de um tema central, as quais se entrelaçam e compõe o assunto.

A técnica de construção de mapas mentais foi desenvolvida pelo inglês Tony Buzan, na última década de 70, logo após constatar que os estudantes que faziam uso de estratégias de trabalho e de anotações diferenciadas, com cores, desenhos, símbolos e ilustrações conseguiam melhores resultados de aprendizagem que os estudantes que não usavam tais métodos, ou seja, a exploração dos hemisférios direito e esquerdo do cérebro no processo de aprendizagem proporcionava melhor absorção do conhecimento passado pelo educador (BOVO \& HERMANN, 2005).

O Mapa Mental ou memograma é uma ferramenta pedagógica de organização de ideias por meio de palavras-chave, cores e imagens em uma estrutura que se irradia a partir de um centro. Os desenhos de mapas mentais beneficiam o aprendizado e, consequentemente, aprimoram a produtividade pessoal. Trata-se de um instrumento de ensino e aprendizagem poderoso e que se sobressai no ensino (BUZAN \& BUZAN, 2009). 


\section{APLICAÇÃO DAS TÉCNICAS DE APRENDIZAGEM ATIVA EM CURSOS DE ENGENHARIA}

A seguir são apresentadas algumas aplicações das técnicas de Aprendizagem Ativa em cursos de engenharia, em particular nos cursos de Engenharia da Computação e de Produção.

\subsection{PROJECT BASED LEARNING (PBL): aplicado à disciplina de Projeto Integrador}

A disciplina de Projeto Integrador é uma unidade curricular integradora, desenvolvida ao longo do semestre, congregando as demais disciplinas do período, com o objetivo de proporcionar ao estudante a construção de conhecimento científico sobre determinada área, por meio do planejamento, organização e execução de proposta acerca de uma situação-problema previamente formulada/escolhida no contexto profissional/empresarial. Um detalhamento da aplicação dessa técnica está descrito em Ferlin \& Oliani (2014 e 2015).

A disciplina de Projeto Integrador tem por objetivo acompanhar e orientar os estudantes no desenvolvimento de um projeto ao longo do semestre letivo. Uma de suas características fundamentais deste projeto é a de ter um caráter multidisciplinar, envolvendo as disciplinas que compõem o período, em que o estudante deve passar por várias etapas do desenvolvimento e o projeto deve incorporar as teorias e conceitos abordados nas disciplinas do período.

Essa atividade é realizada ao longo do semestre e desenvolvida nas Disciplinas de Projetor Integrador, do $1^{\circ}$ ao $8^{\circ}$ período, do Curso de Engenharia da Computação da Faculdade Bagozzi.

Um exemplo de projeto desenvolvido na disciplina de Projeto Integrador I é o projeto "Bengala eletrônica para cegos" (SILVA et al, 2014), que é um dispositivo eletrônico/computacional desenvolvido para auxiliar a locomoção de pessoas com deficiência visual, utilizando sensores ultrassônicos, acelerômetros, e com resposta vibrotátil, mostrado na Figura 1. 
Figura 1 - Foto do projeto bengala eletrônica para cegos

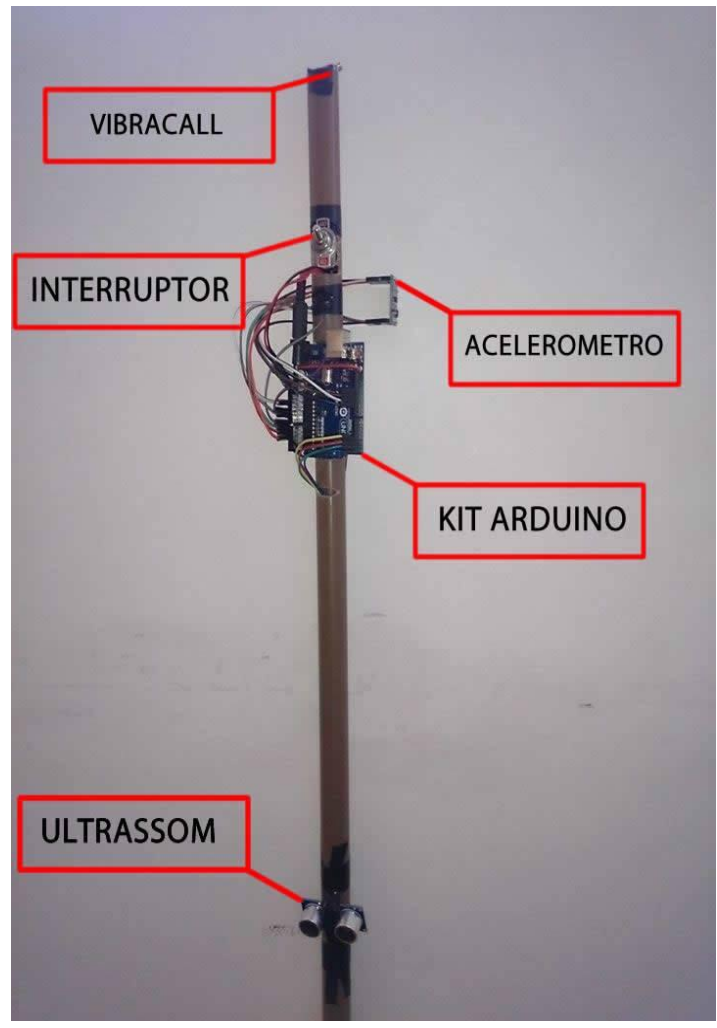

Fonte: SILVA et al, (2014)

\subsection{PROBLEM BASED LEARNING (PBL): aplicação como componente curricular}

A disciplina de PBL consiste em uma unidade curricular do processo de ensinoaprendizagem centrado no estudante como sujeito da aprendizagem e apoiado no professor como facilitador e mediador do processo ensino-aprendizagem. Trabalhando com um problema predefinido, o estudante é guiado a desenvolver a resolução do problema por meio de informações, conceitos e habilidades apreendidas. Entre suas vantagens, se destaca a aplicação do método científico, aquisição de conhecimento e desenvolvimento de habilidades essenciais na resolução de problemas diversos voltados para o mercado de trabalho ou com cunho social. Um detalhamento da aplicação dessa técnica está descrito em Ferlin (2016).

Essa atividade é realizada ao longo do semestre e desenvolvida nas Disciplinas de PBL, do 1ำ ao 8o período, do Curso de Engenharia da Computação do Centro Universitário Uninter.

Um exemplo estudado na disciplina de PBL I (1o Período) é o "Consumo de Bateria de Smartphones em Jogos e Redes Sociais" (FABRI et al, 2017), que consiste em testes de consumo de bateria de smartphones nas condições de uso em jogos e uso de internet acessando redes sociais, mostrado na Figura 2. 
Figura 2 - Gráfico do Teste de descarga de bateria com o jogo SimCity (capacidade mAh x tempo em minutos)

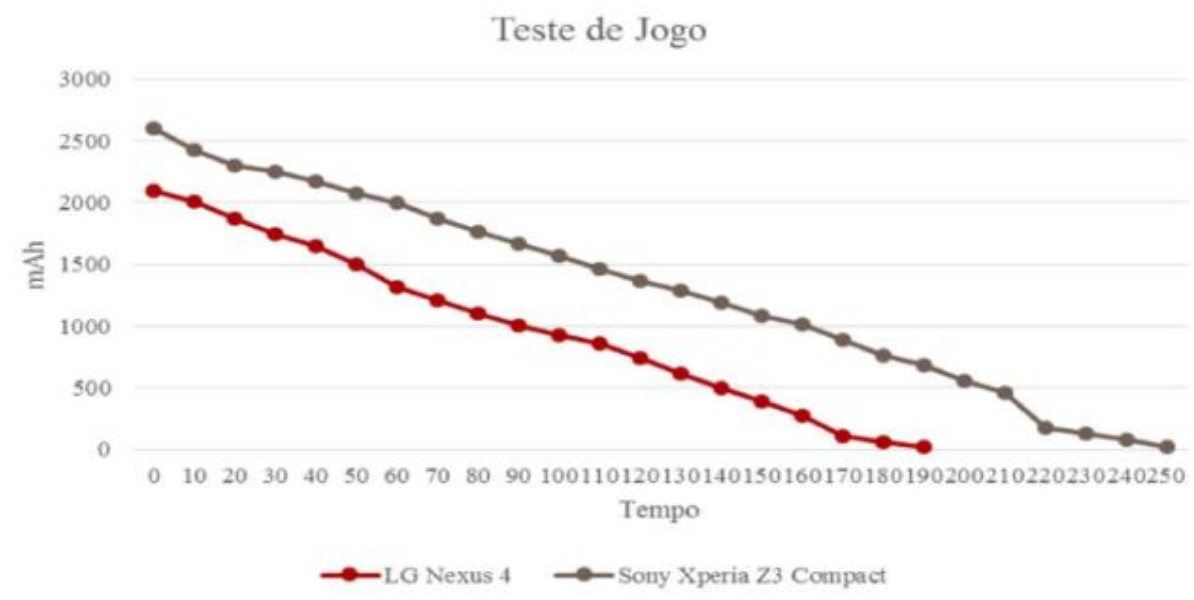

Fonte: FABRI et al, (2017)

\subsection{ESTUDO DE CASO: atividade didática na disciplina de Probabilidade e Estatística}

Essa atividade consiste em propor para os estudantes a elaboração do Estudo de Caso na aplicação dos conceitos de Estatística a um problema real, ao longo do semestre letivo, na Disciplina de Probabilidade e Estatística. Um detalhamento da aplicação dessa técnica está descrito em Ferlin (2020).

O objetivo da atividade é fazer com que os estudantes apliquem os conteúdos da Estatística em problemas reais motivando-os à disciplina e colocando-os no centro do processo de ensino e aprendizagem, por meio de uma técnica da Aprendizagem Ativa.

Essa atividade é realizada ao longo do semestre e desenvolvida na Disciplina de Probabilidade e Estatística, 2o período, do Curso de Engenharia de Produção do Centro Universitário UniDomBosco.

A atividade ocorre na segunda metade da disciplina, basicamente no $2^{\circ}$ bimestre, após a apresentação dos conceitos iniciais do conteúdo de Estatística, pois o objetivo é aplicar os conteúdos a problemas reais, escolhidos pelos estudantes, e, também, o de despertar o interesse pela disciplina.

Os estudos de caso foram realizados em diversos segmentos, como farmacêutica, supermercado, automobilística, indústria, telecomunicação e outras. Abaixo está descrito um exemplo de Estudo de Caso realizados na Disciplina de Probabilidade e Estatística:

Capabilidade da Peca Stator Spacer - O Stator Spacer é parte do conjunto da bomba injetora de um motor a diesel. $O$ estudo foi baseado na mensuração do diâmetro da peça, que possui como medida nominal $\varnothing 8,33$ e com tolerância de \pm $0,05$ ( $\varnothing 8,280$ a $\varnothing 8,380)$. A amostra referiu-se a uma encomenda de teste realizada pela empresa fornecedora da peça Stator Spacer. 


\subsection{MAPA MENTAL: aplicação como atividade didática nas disciplinas}

Essa atividade consiste em propor para os estudantes a elaboração do Mapa Mental sobre um tema proposto na disciplina, logo no começo do semestre letivo, no início da disciplina. Um detalhamento da aplicação dessa técnica está descrito em Ferlin \& Shmeil (2020).

O objetivo da atividade é fazer com que os estudantes tenham um entendimento geral sobre a temática apresentada motivando-os à disciplina e colocando-os no centro do processo de ensino e aprendizagem.

Essa atividade foi aplicada nas disciplinas de Fundamentos de Engenharia, $1^{\circ}$ período, e de Probabilidade e Estatística, 2o período, do Curso de Engenharia de Produção do Centro Universitário UniDomBosco.

A atividade foi realizada nas primeiras aulas das disciplinas, logo após as apresentações iniciais, pois o objetivo era despertar o entendimento geral sobre a temática "Engenharia" no caso da Disciplina de Fundamentos de Engenharia e a temática "Probabilidade" na Disciplina de Probabilidade e Estatística.

$\mathrm{Na}$ Figura 3 é mostrado um exemplo de Mapa Mental na Disciplina de Fundamentos de Engenharia (1ํㅜㄹo Período) do curso de Engenharia de Produção, do Centro Universitário UniDomBosco, com base na temática "Engenharia".

Figura 3 - Exemplo de Mapa Mental

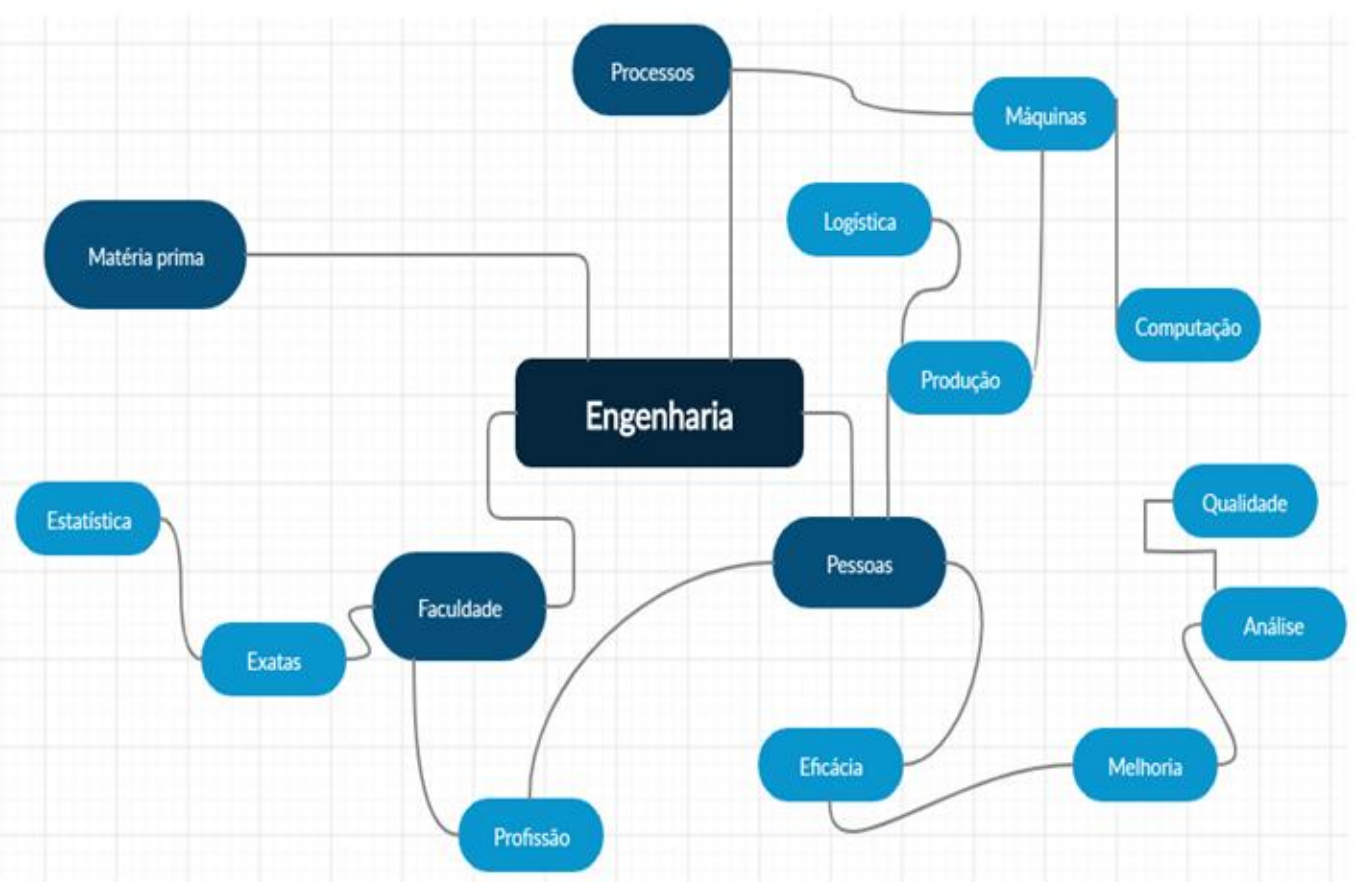

Fonte: Ferlin \& Shmeil (2020)

\section{CONSIDERAÇÕES FINAIS}

As diversas atividades favorecem o aprimoramento do processo de ensino e aprendizagem, por meio da aplicação do conceito de Aprendizagem Ativa. Isto porque os estudantes são agentes ativos nesse processo, desde o momento da pesquisa do caso real a ser aplicado, passando pela elaboração em equipe dos estudos, culminando com a apresentação em sala de aula, além da elaboração documento (artigo, relatório ou monografia). 
Nessas atividades foram fomentados alguns conceitos do soft skills como o trabalho em equipe, comunicação verbal e escrita, e proporcionando autonomia no processo de ensino e aprendizagem.

Dessa forma, o objetivo primário das atividades foi alcançado, pois motivou os estudantes no aprimoramento e na busca do conhecimento, despertando-os no estudo e aplicação dos conceitos das disciplinas em problemas reais.

\section{REFERÊNCIAS}

ANDERSON, L. W.; KRATHWOHL, D.R. A taxonomy for learning, teaching, and assessing. New York: Longman, 2001.

BARBOSA, E. F.; MOURA, D. G. Metodologias ativas de aprendizagem na Educação Profissional e Tecnológica. B. Tec. Senac, Rio de Janeiro, v. 39, n.2, p.48-67, maio/ago. 2013.

BARRET, T. Understanding Problem - based Learning. In: Handbook of enquiry \& problem based learning. Disponível em http://www.nuigalway.ie. 2004.

BLOOM, B.S. (Ed.). Taxonomy of educational objectives: the classification of educational goals: handbook i, cognitive domain. New York: Longman, 1956.

BOVO, V.; HERMANN, W. Mapas mentais - enriquecendo inteligências. Edição dos autores, 2005.

BUZAN, T. Saber pensar. Lisboa: Editorial Presença, 1996.

BUZAN, T.; BUZAN, B. The mind map book: unlock your creativity, boost your memory, change your life the mind map book. Londres: BBC Active, 2009.

ELMOR Filho, G.; et al. Uma nova sala de aula é possível: aprendizagem ativa na educação em engenharia. Rio de Janeiro-RJ: LTC, 2019.

FABRI, C.P.; GONÇALVES, H.R.K.; MACEDO, G.; DE PAULO, L.G.; CICHACZEWSKI, E. Metodologia PBL na Introdução à Engenharia da Computação: Consumo de Bateria de Smartphones em Jogos e Redes Sociais. In: CONTECC 2017 - Congresso Técnico Científico da Engenharia e da Agronomia. Anais. Belém-PA, 2017.

FERLIN, E.P. A Aprendizagem Baseada em Problemas (PBL-Problem Based Learning) Aplicada na Educação em Engenharia: um estudo de caso no Curso de Engenharia da Computação. In: COBENGE 2016 - XLIV Congresso Brasileiro de Educação em Engenharia. Anais. Natal-RN, 2016.

FERLIN, E.P. Aprendizagem ativa nas disciplinas de formação básica de um curso de engenharia: aplicação do estudo de cãs na disciplinas de probabilidade e estatística. In: COBENGE 2020 - XLVIII Congresso Brasileiro de Ensino de Engenharia e III Simpósio Internacional de Educação em Engenharia da Abenge. Anais. Online, 2020.

FERLIN, E.P.; SHMEIL, M..A.H. Utilização das técnicas de aprendizagem ativa na educação em engenharia: estudo de caso dos mapas mentais. In: COBENGE 2020 -

XLVIII Congresso Brasileiro de Ensino de Engenharia e III Simpósio Internacional de Educação em Engenharia da Abenge. Anais. Online, 2020. 
FERLIN, E.P; OLIANI, D. A Disciplina de Projeto Integrador como Elemento Norteador do Processo Ensino-Aprendizagem: a Experiência do Curso de Engenharia da Computação. In: COBENGE 2014 - XLII Congresso Brasileiro de Ensino de Engenharia. Anais. Juiz de Fora-MG, 2014.

FERLIN, E.P; OLIANI, D. Análise da Implantação do Projeto Integrador no Curso de Engenharia da Computação. In: COBENGE 2015 - XLIII Congresso Brasileiro de Ensino de Engenharia. Anais. São Bernardo do Campo-SP, 2015.

GIL, A.C. Como elaborar projetos de pesquisa. 4. ed. São Paulo-SP: Atlas, 2007.

MELO, R.C de. Estratégias de ensino e aprendizagem baseadas em problemas (PBL) no ensino tecnológico. VIII Workshop de Pós-graduação e Pesquisa do Centro Paula Souza. Sistemas produtivos: da inovação à sustentabilidade. ISSN: 2175-1897. 2013.

PILLA JR, V.; FERLIN, E.P. Os níveis de aprendizagem da taxonomia de Bloom aplicados em uma disciplina de um Curso de Engenharia da Computação. In: COBENGE 2010 XXXVIII Congresso Brasileiro de Educação em Engenharia. Anais. Fortaleza-CE, 2010.

PINTO, A.S.S.; et al. Inovação didática - projeto de reflexão e aplicação de metodologias ativas de aprendizagem no ensino superior: uma experiência com "peer instruction". Janus, Lorena, ano 6, n. 15, 1jan./jul., p.75-87, 2012.

PORTILHO, E. Como se aprende? estratégias, estilos e metacognição. Curitiba-PR: Wak Editora, 2009.

REVANS, R. ABC of action learning. Farnham: Gower, 2011.

RIBEIRO, L.R.C. Aprendizagem Baseada Em Problemas (PBL) Na Educação em Engenharia. Revista de Ensino de Engenharia, v. 27, n. 2, p. 23-32. ISSN 0101-5001. 2008.

SILVA, E.; VITALINO, L.; ZANARDI, M.O.; FERLIN, E.P.; CICHACZEWSKI, E. Bengala para Detecção de Obstruções ao Caminhar para uma PCD Visual. In: CRICTE 2014 XXVI Congresso Regional de Iniciação Científica \& Tecnológica em Engenharia. Anais. Alegrete-RS, 2014.

STANFORD UNIVERSITY. Speaking of teaching. Stanford University Newsletter on Teaching, Vol.11, No. 1, 2001.

YEE, K. Interactive techniques. Disponível em: http:// https://www.usf.edu/atle/documents/handout-interactive-techniques.pdf. Acesso em: abril 2019, 2010.

YIN, R.K. Estudo de caso: planejamento e métodos. 5a edição. Porto Alegre-RS: Bookman, 2015. 


\title{
APPLICATION OF ACTIVE LEARNING TECHNIQUES IN ENGINEERING PROGRAMS
}

\begin{abstract}
This paper presents the application of some active learning techniques in engineering courses, specific in computer engineering and production engineering courses, and that could be used in other courses, but not only in engineering. Active learning is a catalyst in the teaching and learning process and its use can result in an improvement of learning by the student, as he is the active element in the process, no longer being a mere spectator and become the main actor. Several techniques can be used and each of them has its characteristics and it is up to the teacher to analyze and use a certain technique that allows the development of competencies in students. The conclusion reiterates the importance of awakening motivation in students and placing them as active elements in the teaching and learning process.
\end{abstract}

Keywords: Engineering, Education, Active Learning, Learning Levels. 